\title{
Un dispositif bien encadré
}

\section{Gudrun Bogdanski}

Traducteur : Dominique Gélin

\section{OpenEdition}

\section{Journals}

Édition électronique

URL : http://journals.openedition.org/ries/3267

DOI : 10.4000/ries.3267

ISSN : 2261-4265

Éditeur

Centre international d'études pédagogiques

Édition imprimée

Date de publication : 1 septembre 1996

Pagination : $35-43$

ISSN : 1254-4590

Référence électronique

Gudrun Bogdanski, «Un dispositif bien encadré », Revue internationale d'éducation de Sèvres [En ligne], 11 | 1996, mis en ligne le 01 octobre 2013, consulté le 01 mai 2019. URL : http://

journals.openedition.org/ries/3267; DOI : 10.4000/ries.3267

Ce document a été généré automatiquement le 1 mai 2019

(c) Tous droits réservés 


\section{Un dispositif bien encadré}

\section{Gudrun Bogdanski}

Traduction : Dominique Gélin

1 L'Allemagne étant une République à structure fédérale, l'évaluation des élèves est pratiquée de façon différente dans chaque Land, néanmoins une façon identique de voir les choses se dégage sur les points essentiels. L'exemple choisi ici est celui de la RhénanieDu-Nord-Westphalie en étudiant, d'un côté, le cadre juridique de l'évaluation des résultats des élèves et de l'autre, les pratiques dans le domaine plus spécifique des cours de français des premier et second cycles de l'enseignement secondaire.

On sait que le système scolaire n'est pas le même dans les seize Länder, étant donné la structure fédérale de la République. Non seulement les types d'écoles mais également la durée du curriculum diffèrent. Dans les nouveaux Länder, à l'exception du Brandenbourg, on peut passer l'Abitur à la fin de la douzième année, alors qu'il faut treize ans ailleurs. De la même façon, l'Abitur est centralisé en Bavière et dans le Bade-Wurtemberg mais décentralisé dans les autres Länder.

Dans quelques Länder seulement, le cycle de l'enseignement primaire s'étend sur six années; dans la plupart, comme la Rhénanie-du-Nord- Westphalie, il n'en comporte que quatre. La Conférence permanente des ministres de l'Éducation qui se réunit quatre fois par an et qui ne peut prendre de décision qu'à l'unanimité, veille cependant à l'unité du système éducatif, indispensable si l'on veut maintenir une reconnaissance réciproque des diplômes sur tout le territoire de la République fédérale. Il n'est pas possible toutefois, dans le cadre de cette étude de faire le tour des différentes pratiques évaluatives dans les seize Länder.

Décrire les pratiques d'évaluation en Rhénanie-Du-Nord-Westphalie, qui est le Land le plus peuplé, permet de dégager, à la fois, les nombreux points communs et les différences, nettement marquées, avec les autres Länder. 


\section{Le cadre juridique}

5 En Rhénanie-Du-Nord-Westphalie, l'évaluation des résultats obtenus par les élèves est définie par ce qu'on appelle l'Allgemeine Schulordnung de $1978^{1}$, dispositif juridique qui prend appui sur la Constitution du Land et auquel toutes les écoles publiques ainsi que les écoles privées reconnues par le ministère, depuis l'école élémentaire jusqu'au second cycle de l'enseignement secondaire, sont soumises. Ces instructions déterminent toutes les relations, sur le plan juridique, entre élèves, parents et enseignants. Le chapitre intitulé «Évaluation des résultats obtenus par les élèves et passage dans la classe supérieure » qui traite de toutes les questions afférentes, pour tout type d'école, nous intéresse directement.

Quelques-unes de ces instructions, importantes, et qui concernent plus précisément les pratiques d'évaluation, vont être explicitées ci-dessous.

7 Tous les résultats obtenus par les élèves sont évalués à l'aide d'une note chiffrée, à l'exception des deux premières années de l'école élémentaire, au cours desquelles les résultats obtenus par les élèves font l'objet non pas d'une notation mais d'une appréciation argumentée. L'échelle des notes va de 1 (très bien) - qui est la meilleure note - à 6 (insuffisant), la plus mauvaise; à l'intérieur de cette fourchette on trouve 2 (bien), 3 (satisfaisant), 4 (passable) et 5 (médiocre). La note varie selon que le travail satisfait plus ou moins bien aux exigences d'une discipline. La note la plus élevée, «très bien ", par exemple, ne signifie pas que le travail de l'élève ne comporte aucune faute, mais qu'il répond remarquablement bien à ces exigences.

8 La plus mauvaise note, « insuffisant », ne correspond pas, elle non plus, à un manque total de connaissances ; elle signifie que le travail fourni par l'élève ne correspond pas à ce qui est exigible et même que le manque de connaissances fondamentales est tel qu'il ne pourra être comblé dans un avenir plus ou moins rapproché. Toutes les notes sont attribuées en fonction de ces exigences.

9 Les notes qui correspondent aux différents travaux, à l'oral et à l'écrit, sont réunies pour aboutir aux moyennes inscrites dans les bulletins des élèves : ils reçoivent deux bulletins par année scolaire, le premier en janvier, à la fin du premier semestre, et le deuxième à la veille des vacances d'été. La fin de l'année scolaire peut être fixée à des dates très variables, selon la réglementation des différents Länder, au mois de juin, juillet ou août. Dans le bulletin de fin d'année figure la décision de passage dans la classe supérieure. Celui-ci dépend des notes obtenues au cours du second semestre. Si un élève a de mauvaises notes dans plusieurs disciplines, il n'est pas admis dans la classe supérieure. La réglementation définit de manière très stricte les conditions de passage en classe supérieure. Une mauvaise note dans une discipline peut être compensée par une meilleure note dans une autre discipline. Un élève peut, à certaines conditions, subir un examen de passage après les vacances d'été, afin d'être, malgré tout, admis dans la classe supérieure.

10 À la fin de chaque cycle, ce sont les notes figurant dans le bulletin qui déterminent le cursus que va suivre l'élève. C'est ainsi que sera prise, à la fin du cycle élémentaire, la décision d'orienter l'élève vers l'un des quatre types d'école suivants, correspondant à quatre niveaux d'exigences différents : Hauptschule ${ }^{2}$ (de la $5^{\mathrm{e}}$ à la $10^{\mathrm{e}}$ année), Realschule (de la $5^{\mathrm{e}}$ à la $10^{\mathrm{e}}$ année), Gymnasium ${ }^{4}$ (de la $5^{\mathrm{e}}$ à la $13^{\mathrm{e}}$ année) et Gesamtschule ${ }^{5}$ (de la $5^{\mathrm{e}}$ à la $13^{\mathrm{e}}$ 
année). Tandis que les trois premiers types d'école ont une longue tradition derrière eux, le nombre d'écoles du quatrième type - la Gesamtschule - n'a commencé à croître qu'à partir des années soixante-dix. La Gesamtschule procède, volontairement, d'une manière différente de celle des trois autres types traditionnels d'école en ce qui concerne l'évaluation et le passage dans la classe supérieure.

Dans la Gesamtschule, des élèves ayant des niveaux de compétence différents sont répartis dans des groupes de niveaux. On trouve là, réunis, les élèves des trois types d'école traditionnels. Tous les élèves d'une Gesamtschule sont certes admis dans la classe supérieure indépendamment du niveau de compétence atteint, mais à la fin du premier cycle de l'enseignement secondaire, ils obtiennent des diplômes de fin d'études différenciés, selon le niveau des groupes dans lesquels ils se trouvaient et les notes qu'ils y ont obtenues : le diplôme de la Hauptschule, celui de la Realschule (Fachoberschulreife) ou la qualification équivalente à celle qui permet, au Gymnasium, de passer de $10^{\mathrm{e}}$ en $11^{\mathrm{e}}$ année. Il convient d'ajouter que conformément au principe des passerelles, les trois diplômes ci-dessus peuvent être obtenus dans les trois types traditionnels d'école. Les élèves qui ne fréquentent pas le premier cycle de l'enseignement secondaire du Gymnasium, mais ont obtenu dans le type d'école choisi par eux, Hauptschule, Realschule ou Gesamtschule, une qualification particulièrement bonne, peuvent entrer en première année du second cycle de l'enseignement secondaire, au Gymnasium, s'ils désirent se présenter à l'Abitur ou au Fachhochschulreife qui leur donnera aussi accès aux études supérieures. Ces deux diplômes, qui leur donnent le droit d'entrer à l'université ou dans une Fachhochschule pour y poursuivre des études, peuvent être obtenus au Gymnasium, dans une Gesamtschule ou dans un établissement d'enseignement professionnel ${ }^{6}$.

Ces rapides exemples montrent que le système scolaire en Rhénanie-Du-Nord-Westphalie est, d'une part, très complexe mais, d'autre part, ouvert, avec de nombreuses possibilités de passerelles.

Les Gymnasien existent dans tous les Länder, mais les autres types d'école peuvent y prendre des aspects différents. Dans le second cycle du secondaire, les notes de la $12^{\mathrm{e}}$ et de la $13^{e}$ année, c'est-à-dire des deux dernières années, sont déterminantes pour obtenir l' Abitur. Ces deux années sont considérées comme une phase de qualification dont les notes comptent pour les $2 / 3$ dans le bulletin du candidat à l'Abitur; un tiers seulement des notes dépend des examens écrits et oraux.

Sur le bulletin du candidat à l'Abitur, les notes sont converties en points. Pour la note la plus haute - très bien - par exemple, on attribue un 15 , un 14 , ou un 13 , selon la teneur du bulletin ; pour la plus basse - insuffisant - un 0 . L'accès aux études supérieures, dans les disciplines soumises à un numerus clausus, dépendra de la moyenne des notes de ce bulletin. Les enseignants qui attribuent ces notes portent une responsabilité toute particulière: ce sont ces notes qui donneront, ou non, la possibilité après l'Abitur d'étudier ou de bénéficier d'une formation. En dehors des études de médecine, auxquelles on n'a accès qu'après avoir passé un test d'admission, en plus de l'Abitur, il n'y a pas d'autres examens d'entrée dans le supérieur qui puissent compenser les notes obtenues à l'Abitur.

15 L'Allgemeine Schulordnung ne précise pas dans le détail comment un enseignant doit évaluer les résultats des élèves. Elle définit, par contre, les critères qu'il faudra observer 
pour cette évaluation. Un enseignant devra, pour l'essentiel, respecter les principes suivants :

- le travail d'un élève pendant le cours aura autant de valeur que son travail lors des contrôles écrits. Les compétences que l'on attend d'un élève dans chaque discipline doivent apparaître, très concrètement, dans le programme de cette discipline, comme on le montrera dans la deuxième partie de cette étude, à propos de l'enseignement du français ;

- un enseignant à le devoir d'indiquer à un élève, à chaque fois qu'il le désire, à quel niveau de compétences il se situe ;

- lorsqu'il s'agit d'évaluer les résultats des élèves, l'enseignant dispose d'une certaine marge d'appréciation, pour laquelle il est seul compétent; personne, ni le directeur, ni ses collègues ni l'administration n'a le droit d'intervenir. Un élève, ou ses parents, peut, bien entendu, contester une note. Si la chose lui paraît justifiée, le professeur qui enseigne dans la discipline peut modifier cette note. S'il ne veut pas le faire, la contestation, jointe à un rapport de l'enseignant, où il justifie son évaluation, est envoyée à l'administration. C'est là qu'on déterminera si la note a été attribuée à juste titre, en respectant les principes de l'évaluation, ou si elle doit être relevée.

Le nombre et la durée des contrôles écrits sont fixés, pour toutes les matières, par un décret ministériel. L'Allgemeine Schulordnung prescrit dans quelles conditions ces contrôles peuvent être mis en place et évalués. On trouve des informations concrètes et des modèles de devoirs à donner aux élèves dans les programmes des différentes disciplines.

On établit une distinction entre ce qu'on appelle les disciplines " écrites ", où il y a des contrôles écrits et celles où il n'y en a pas, disciplines "non écrites ». Dans le premier cycle du secondaire, l'allemand, les mathématiques, les première et deuxième langues étrangères sont des disciplines " écrites ", pour tous les élèves. Dans le deuxième cycle du secondaire, c'est l'élève lui-même qui détermine d'après certains critères, mais aussi en fonction de ses vœux personnels, dans quelles matières il aura des contrôles écrits, auxquels il devra alors se soumettre.

Le nombre des contrôles écrits dépend, dans le premier cycle du secondaire, du nombre d'heures pendant lesquelles la matière est enseignée dans la semaine. On rédigera, par exemple, quatre contrôles dans une discipline où il y a quatre heures de cours par semaine. Dans le second cycle du secondaire, au Gymnasium, il n'y aura de toute façon que deux contrôles par semestre.

19 Au début du premier cycle du secondaire, les contrôles écrits durent entre une et deux heures de cours (de 45 à 90 minutes); en $9^{\mathrm{e}}$ et $10^{\mathrm{e}}$ année entre une et trois heures de cours, selon les matières (de 90 à 135 minutes). Dans le second cycle du secondaire, les contrôles durent entre deux et quatre heures (de 90 à 180 minutes), pour les disciplines où il y a trois heures de cours par semaine, et entre deux et six heures de cours (de 90 à 270 minutes) dans les disciplines où il $\mathrm{y}$ a six heures de cours par semaine.

On ne peut donner qu'un contrôle écrit par jour aux élèves, afin qu'ils ne soient pas trop surchargés de travail. Dans le premier cycle du secondaire, on ne peut pas donner plus de deux contrôles par semaine, dans le second cycle pas plus de trois. Étant donné la durée de certains contrôles, ce souci de ne pas surcharger les élèves est compréhensible. Les contrôles doivent être répartis le plus régulièrement possible tout au long de l'année scolaire, ce qui suppose de la part de l'enseignant une planification à long terme. Pour donner aux élèves la possibilité de se préparer à ces contrôles en faisant les révisions 
nécessaires, les contrôles «surprises » ne sont pas autorisés; ils doivent, au contraire, être toujours annoncés suffisamment longtemps à l'avance.

21 Le niveau exigé, lors de ces contrôles écrits, doit correspondre au niveau de compétence moyen d'une classe ou d'un groupe de niveau, ce qui signifie qu'un contrôle, auquel un tiers des élèves n'a pas obtenu de résultats suffisants, doit être soumis à l'approbation du directeur de l'établissement. C'est lui qui décide si le contrôle peut faire partie de l'évaluation ou s'il faut en faire un autre.

\section{Un exemple d'évaluation : l'enseignement du français}

Le chapitre «Résultats obtenus par les élèves et évaluation» du programme d'enseignement du français dans le premier cycle de l'enseignement secondaire aborde les différents aspects de l'évaluation de l'écrit, de ce qu'on appelle les Klassenarbeiten (contrôles écrits), puis les différentes façons dont les élèves participent au cours.

Le professeur doit choisir, parmi les différents types de contrôles proposés dans le programme, ceux qui sont adaptés à sa classe et au niveau atteint dans la perspective d'un enseignement fondé sur les besoins de l'élève. Cet enseignement doit inciter les élèves à être actifs, autonomes, créatifs dans la langue étrangère. En cours et, ensuite, lors du contrôle qui servira à tester les acquis, les tâches de production où ils s'expriment directement dans la langue étrangère jouent un rôle important.

On distingue trois types de tâches : fermées, semi-ouvertes et ouvertes. Le premier type de tâche, traditionnel, dans lesquels les élèves répondent aux mêmes questions, sert à contrôler leurs connaissances en grammaire, vocabulaire et leurs compétences dans les deux domaines de la compréhension de l'oral et de l'écrit. Ce genre de tâche occupe une place prépondérante en début d'année. Dictées et traductions sont expressément exclues lors des contrôles. Dans le type semi-ouvert, l'élève qui s'exprime directement dans la langue étrangère est guidé : il doit, par exemple, compléter un dialogue, décrire des images connues. Le type ouvert offre la plus grande liberté d'expression personnelle à l'élève. Ce genre de tâche doit permettre de développer l'imagination et l'activité langagière autonome de l'élève ; il s'agit, par exemple, d'inventer une histoire à partir de mots et d'idées stimulants ou de rédiger un compte rendu de lecture sous forme de lettre.

Les contrôles doivent dans leur ensemble être variés afin de tenir compte des différents profils d'élèves et des différents contenus du cours. Les tâches confiées aux élèves doivent auparavant faire l'objet d'un entrainement approfondi en cours. Les tâches du type semiouvert et ouvert doivent faire leur apparition aussi tôt que possible dans le cours, bien entendu sous une forme simple, afin que les élèves y soient entrânés dès le départ. Cela exige de la part de nombreux enseignants, qui donnaient jusque là des tâches aux élèves dans le but principalement de contrôler leurs connaissances en vocabulaire, en grammaire et en orthographe, qu'ils changent leur façon de voir les choses et leur pratique.

Cela signifie également un surcroît de travail en ce qui concerne les corrections et leur évaluation: les contrôles où il suffit de distinguer entre réponses correctes et réponses erronées sont plus faciles et plus rapides à corriger que les contrôles où chaque individu propose une réponse personnelle pour laquelle il faut évaluer la correction de la langue, la qualité de l'expression et le contenu. La nature et la gravité des fautes de langue dans les contrôles écrits doivent être indiquées en marge du texte de l'élève. Il faut utiliser, 
pour cela, une certaine codification des fautes. La note du contrôle écrit est justifiée par une appréciation argumentée qui fait apparaitre les points forts et les faiblesses du travail de l'élève et qui porte sur la correction de la langue, la qualité de l'expression et le contenu. Lorsqu'il rend les travaux aux élèves, le professeur doit obligatoirement expliquer quels sont ses critères d'évaluation.

Pour la participation en cours, qui a la même importance pour l'attribution de la note dans le bulletin que les résultats des contrôles écrits, on évalue la qualité et la continuité des interventions des élèves, à l'oral et à l'écrit. Ici la participation orale, par exemple la participation de l'élève au dialogue durant le cours, les interventions dans le contexte des exercices et des jeux de rôles, de courts exposés, tout cela joue un rôle, parallèlement aux devoirs à la maison, écrits et oraux, ainsi que ce qu'on appelle les schriftliche Übungen (exercices écrits). Ces derniers demandent une petite explication car ce type de contrôle est strictement défini par l'Allgemeine Schulordnung: ces exercices écrits courts (d'une durée maximale de 15 minutes) ne peuvent avoir lieu qu'occasionnellement, ils doivent, en règle générale, être annoncés à l'avance, de la même façon que les contrôles écrits, et ne portent que sur ce qui a été traité lors du cours précédent.

Étant donné l'importance de la participation des élèves en cours pour l'évaluation des résultats, l'enseignant doit sans cesse susciter la créativité des élèves. Cette créativité doit faire l'objet de sa part d'une observation attentive. Si les résultats obtenus dans la participation au cours sont meilleurs que les résultats des contrôles écrits, les notes de participation peuvent servir à compenser de mauvais résultats aux contrôles écrits.

Inversement, de bons résultats à l'écrit ne peuvent suffire à eux seuls pour obtenir une bonne note sur le bulletin. Il faut que d'autres résultats, correspondant à des activités variées, viennent s'y ajouter de façon continue à l'écrit et à l'oral.

Pour tenir compte des progrès effectués par les élèves dans le second cycle de l'enseignement secondaire, d'autres types de contrôles sont utilisés, en dehors de ceux qui viennent d'être exposés. Ces contrôles doivent préparer les élèves à leur examen, les amener au niveau d'exigence de l'Abitur écrit et oral. Lors de cet examen, les élèves doivent analyser un texte authentique inconnu, guidés pour ce faire par quatre à six questions appelées «sujets d'étude ». Ce texte correspond aux thèmes abordés en $11^{\mathrm{e}}$ et $12^{\mathrm{e}}$ année, si bien que les élèves peuvent réutiliser à l'examen, dans leur analyse, les connaissances acquises en cours.

31 Dans la première partie, orale, de l'Abitur l'élève analyse également un texte inconnu devant un jury; dans la deuxième partie, on aborde des domaines plus larges de la discipline étudiée. l'Abitur étant un examen décentralisé, il convient de noter que l'envoi et le choix de sujets d'examen par les enseignants spécialistes de la discipline et l'administration représentent un travail considérable. Les enseignants doivent, lorqu'ils font leurs propositions de sujets d'examen, donner des indications très concrètes sur le contenu de leur cours et le niveau d'exigence attendu des élèves.

Le ministère du Land choisit les sujets d'examen pour chaque établissement, parmi des propositions qui doivent rester secrètes. Dans les deux Länder où l'Abitur est centralisé, la Bavière et le Bade-Wurtemberg, tous les élèves ont les mêmes sujets.

Les élèves du second cycle de l'enseignement secondaire, au Gymnasium, s'entraînent à l'analyse de texte lors de contrôles écrits, afin de se préparer à l'Abitur. La correction s'effectue selon les mêmes critères que dans le premier cycle de l'enseignement secondaire : évaluation du contenu et de la correction de la langue ainsi que la qualité de 
l'expression, codification des fautes selon leur nature et leur gravité, justification de la note dans une appréciation argumentée faisant apparaitre les points forts et les faiblesses du travail de l'élève. Comme les élèves ont, dans ce cycle, un niveau plus élevé, la correction se fait naturellement de manière plus différenciée que dans le premier cycle et demande beaucoup plus de temps, en raison de la longueur des travaux rendus. L'évaluation de la participation au cours se fonde, comme dans le premier cycle, sur la participation orale au dialogue qui s'instaure pendant le cours, sur les devoirs à la maison et les exercices écrits. À quoi on peut ajouter des exposés et des comptes rendus de cours dans la langue étrangère. Tous les types de contrôles prévus doivent obligatoirement être annoncés par le professeur dès le début de l'année. Dans le second cycle de l'enseignement secondaire c'est donc, là aussi, la continuité du travail qui est déterminante pour l'attribution de la note dans la discipline. Tandis que dans le premier cycle la participation au cours n'est notée qu'à la fin du semestre, l'élève du second cycle est noté deux fois par semestre, dans chaque discipline. Le fait de recevoir suffisamment à temps une information sur le niveau qu'il a atteint, donne à l'élève la possibilité d'améliorer ses résultats au cours du second semestre. En outre toutes les notes qui figureront sur le bulletin doivent obligatoirement avoir été communiquées auparavant oralement à l'élève.

Contrairement aux autres contrôles écrits, les épreuves de l'Abitur ne sont pas corrigées par le seul professeur de la discipline; il y a une double correction. Si le premier et le deuxième correcteur ne parviennent pas à se mettre d'accord, c'est un troisième correcteur qui décide de la note. Cette disposition est valable pour tous les Länder à l'exception du Bade-Wurtemberg, où les épreuves de l'Abitur sont corrigées de façon anonyme, comme en France. Le jury d'oral de l'Abitur est composé des membres suivants : un examinateur, enseignant spécialiste de la discipline, une personne qui établit le procés-verbal, un assesseur, également spécialiste de la discipline, et un président. Le jury doit se mettre d'accord sur la note à attribuer.

\section{L'évaluation soumise au banc d'essai}

Pour celui qui découvre de l'extérieur cette évaluation, telle qu'elle est pratiquée en Rhénanie-Du-Nord-Westphalie, il s'agit là d'une structure complexe, comportant de nombreux règlements. Si l'on veut se livrer à un examen critique, il faut bien garder à l'esprit que l'évaluation des résultats obtenus par les élèves se déroule de façon aussi ouverte que le cours lui-même, organisée de la façon la plus ouverte possible et que, malgré la complexité de la structure, elle doit offrir au plus haut degré toute garantie de transparence et de comparabilité. Toutes ces dispositifs, obligatoires, sont le prix de la liberté.

L'Abitur centralisé, tel qu'il se pratique en Bavière et au Bade- Wurtemberg, a bien entendu en Rhénanie-Du-Nord-Westphalie aussi ses partisans : ils estiment qu'un examen organisé de manière centralisée est plus objectif que ce système décentralisé. Un Abitur centralisé permettrait aux enseignants de la spécialité et à l'administration de faire l'économie d'une quantité non négligeable de travail. Les partisans de l'Abitur décentralisé sont cependant majoritaires dans le Land en raison de la grande liberté d'organisation dont disposent les enseignants spécialistes de la discipline.

On discute actuellement pour savoir comment il serait possible de décharger les enseignants d'une partie du travail, considérable, que représentent les corrections. 
Certains ont proposé de demander aux élèves de faire des travaux plus courts et moins nombreux. Mais il a été objecté à cela qu'une telle réduction accroîtrait la pression du système sur les élèves, obligés d'obtenir un nombre suffisant de résultats satisfaisants tout en ayant moins souvent la possibilité de s'entraîner.

On se demande, dans le cadre de la préparation des nouveaux programmes du second cycle de l'enseignement secondaire qui doivent être publiés en 1998, s'il ne faudrait pas cesser de privilégier, unilatéralement, les analyses de texte au profit d'épreuves plus créatrices, telles qu'elles sont expérimentées à l'heure actuelle dans le premier cycle de l'enseignement secondaire.

\section{NOTES}

1. II s'agit d'instructions, rédigées par des juristes, qui réglementent le fonctionnement général de tous les établissements scolaires, depuis la vie scolaire jusqu'à l'évaluation. Ces textes existent dans tous les Länder. Ce sont des textes de référence en cas de litige. (NdT).

2. École secondaire générale ( $N d l R$.$) .$

3. École moyenne (NdlR.).

4. Lycée (NdlR.).

5. École polyvalente (NdlR)

6. II s'agit d'un établissement qui accueille les apprentis (système dual). (NdT)

\section{RÉSUMÉS}

La Rhénanie-du-Nord-Westphalie sert de cadre pour présenter les règles officielles qui régissent l'évaluation scolaire en Allemagne. L'auteur étudie plus particulièrement les pratiques des enseignants de français, que leur évaluation soit quotidienne, dans la classe, ou certificative, en vue de l'obtention de l'Abitur.

\section{INDEX}

Index géographique : Allemagne, Rhénanie du Nord-Westphalie

Mots-clés : enseignant, évaluation, évaluation de l'élève 
AUTEURS

GUDRUN BOGDANSKI

Inspectrice de langues vivantes, Düseldorf, Allemagne 